University of Nebraska - Lincoln

DigitalCommons@University of Nebraska - Lincoln

2013

\title{
Use of Attosecond Electron Pulses to Image Electronic Motion in Atoms and Molecules
}

Hua-Chieh Shao

University of Nebraska-Lincoln, hshao@unl.edu

Anthony F. Starace

University of Nebraska-Lincoln, astarace1@unl.edu

Follow this and additional works at: https://digitalcommons.unl.edu/physicsstarace

Shao, Hua-Chieh and Starace, Anthony F., "Use of Attosecond Electron Pulses to Image Electronic Motion in Atoms and Molecules" (2013). Anthony F. Starace Publications. 200.

https://digitalcommons.unl.edu/physicsstarace/200

This Article is brought to you for free and open access by the Research Papers in Physics and Astronomy at DigitalCommons@University of Nebraska - Lincoln. It has been accepted for inclusion in Anthony F. Starace Publications by an authorized administrator of DigitalCommons@University of Nebraska - Lincoln. 


\title{
Use of Attosecond Electron Pulses to Image Electronic Motion in Atoms and Molecules
}

\author{
Hua-Chieh Shao and Anthony F. Starace \\ Department of Physics and Astronomy, The University of Nebraska, Lincoln, \\ Nebraska 68588-0299, USA
}

\begin{abstract}
We investigate theoretically the direct imaging of coherent electronic motion in atoms and molecules using attosecond electron pulses. The theories of time-resolved ultrafast electron diffraction and $(e, 2 e)$ momentum spectroscopy as well as the requisite conditions for carrying out time-resolved measurements to obtain timedependent images are discussed. Results of simulations showing images of the motions of coherent superposition states in both the hydrogen atom and the hydrogen molecular ion are shown, thus indicating the capability of ultrafast electron pulses to investigate time-dependent electron dynamics.
\end{abstract}

Keywords: Ultrafast electron diffraction, $(e, 2 e)$ momentum spectroscopy, Attosecond electron dynamics, Timedependent scattering, Imaging electron motion

\section{INTRODUCTION}

Owing to experimental advances in the techniques for producing ultrashort light and ultrashort electron pulses coupled with the important role that electrons play in various kinds of reactions on attosecond time scales, attosecond science ${ }^{1,2}$ has emerged as an important new discipline. Its aim is to investigate electron dynamics on its natural time scale, which involves demanding requirements on the necessary spatial and temporal resolutions. At present ultrafast electron diffraction and microscopy provide sub-Angstrom resolution to study atomic and molecular structures in tabletop scale experiments. ${ }^{3-5}$ Moreover, temporal resolutions have been substantially improved over the past decade in pump-probe setups. ${ }^{5,6}$ Reaction time constants and intermediate molecular structures along a reaction path have been directly imaged ${ }^{7}$ providing detailed information about reaction mechanisms. Incorporating the dimension of time, four-dimensional electron tomography has enabled the reconstruction of different modes of carbon-nanotube motion. ${ }^{8}$ In addition, the ability to generate single-electron pulses further reduces the electron pulse length and improves electron pulse coherence, since space-charge effects are absent. The capabilities of such single-electron pulses to image structures on the atomic scale ${ }^{9}$ and to study time-dependent phenomena ${ }^{5}$ have been demonstrated.

Recently methods for generating attosecond electron pulses have been proposed, ${ }^{10-13}$ and their use for studying time-dependent electron dynamics in atoms and molecules have been simulated. ${ }^{14-17}$ Thus the direct imaging of electronic motion during the course of a reaction by means of attosecond electron pulse probes appears feasible. Here we summarize our recent works ${ }^{16-18}$ concerning the imaging of electronic dynamics using such ultrafast electron pulses. We have investigated theoretically both ultrafast electron diffraction ${ }^{16,18}$ and ultrafast electrom impact ionization ${ }^{17}$ processes, which provide different kinds of information about electronic motion in coordinate space and momentum space respectively. For the case of ultrafast electron diffraction, two different theories ${ }^{16,18}$ are discussed and compared. These are presented in Secs. 2 and 3 respectively. The theory of ultrafast electron momentum spectroscopy is presented in Sec. 4. Our conclusions are presented in Sec. 5.

\section{ULTRAFAST ELECTRON DIFFRACTION AS POTENTIAL SCATTERING}

Our proposed setup for using ultrafast electron diffraction to image electronic motion is shown in Figure 1. The electron dynamics is initiated by some optical pump laser, and it is probed by a time-delayed electron pulse. The electronic motion can be inferred by a series of such time-resolved diffraction images for various time delays.

Further author information: (Send correspondence to A. F. S.)

A. F. S.: E-mail: astarace1@unl.edu, Telephone: 14024722795

H.-C. S.: E-mail: hcshao@huskers.unl.edu, Telephone: 14024725094

Ultrafast Imaging and Spectroscopy, edited by Zhiwen Liu, Proc. of SPIE Vol. 8845, $884504 \cdot$ ㄷ 2013 SPIE $\cdot$ CCC code: $0277-786$ X/13/\$18 · doi: $10.1117 / 12.2023522$ 


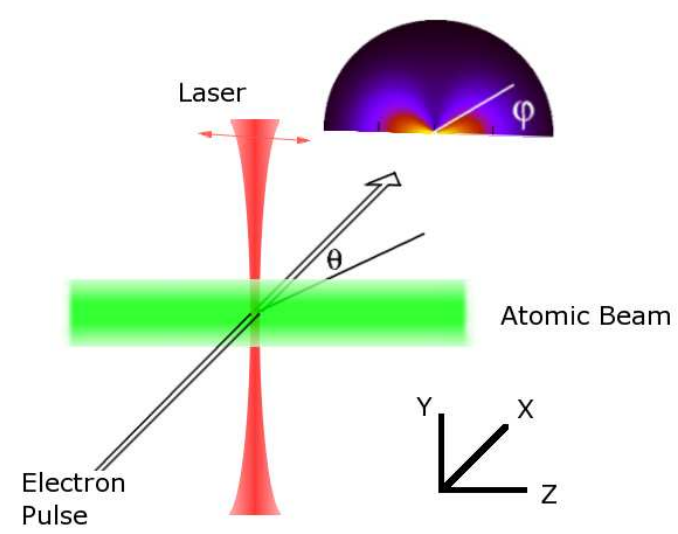

Figure 1: Schematic experimental setup for ultrafast electron diffraction from a coherent superposition of target states. ${ }^{16}$ The coherent state is produced by an optical pump pulse and probed by a time-delayed ultrafast electron pulse. The diffraction images are measured as a function of pump-probe delay time. The red arrow indicates the polarization direction of the pump laser. For future reference, the scattering angle $\theta$ and the azimuthal angle $\varphi$ are defined here.

The conventional theory of high energy electron diffraction formulates it as elastic electron scattering from a potential representing the target. ${ }^{19}$ In the following we sketch the theory and provide simulations in which coherent states of an atom and a molecule are the targets.

\subsection{Theory}

Consider first an atomic target. The effective potential between the projectile electron and the target (located at the origin) is the combination of the nuclear Coulomb potential and the potential produced by the electron density $\rho(\boldsymbol{x})$, namely,

$$
V_{\mathrm{eff}}=-\frac{Z}{\left|\boldsymbol{x}_{0}\right|}+\int d \boldsymbol{x}^{\prime} \frac{\rho\left(\boldsymbol{x}^{\prime}\right)}{\left|\boldsymbol{x}_{0}-\boldsymbol{x}^{\prime}\right|},
$$

where $\boldsymbol{x}_{0}$ is the coordinate of the incident projectile electron, $Z$ is the atomic number of the atom, and where Gaussian units, $e=1$, and $\hbar=1$ are used throughout this article. The electron density is defined as

$$
\rho(\boldsymbol{x})=\int d\left\{\boldsymbol{r}_{i}\right\}\left|\psi_{1}\left(\left\{\boldsymbol{r}_{i}\right\}\right)\right|^{2} \sum_{i=1}^{Z} \delta\left(\boldsymbol{x}-\boldsymbol{r}_{i}\right),
$$

where $\psi_{1}$ is the target electronic wave function, $\left\{\boldsymbol{r}_{i}\right\}$ schematically denotes the collection of coordinates of the target electrons, and $\delta$ is the Dirac delta function.

The wave function $\psi_{0}$ of the projectile electron in the asymptotic region $\left(x_{0} \gg 1\right.$, where $\left.x_{0} \equiv\left|\boldsymbol{x}_{0}\right|\right)$ is the sum of an incident plane wave and a scattered spherical wave, i.e.,

$$
\psi_{0} \sim e^{i \boldsymbol{k}_{0} \cdot \boldsymbol{x}_{0}}+f\left(\hat{\boldsymbol{k}}_{a}\right) \frac{e^{i k_{a} x_{0}}}{x_{0}},
$$

where $f$ is the scattering amplitude, $\boldsymbol{k}_{0}$ and $\boldsymbol{k}_{a}$ are the respective momenta of the electron before and after the collision, and $\hat{\boldsymbol{k}}_{a}$ symbolizes the scattering angles of the electron. In the first-order Born approximation, the scattering amplitude of the projectile electron is ${ }^{19}$

$$
f\left(\hat{\boldsymbol{k}}_{a}\right)=-\frac{2 \mu}{\boldsymbol{s}^{2}}\left(Z-\int d \boldsymbol{x}^{\prime} e^{i \boldsymbol{s} \cdot \boldsymbol{x}^{\prime}} \rho\left(\boldsymbol{x}^{\prime}\right)\right),
$$


where $\mu$ is the reduced mass of the collision system, and $\boldsymbol{s} \equiv \boldsymbol{k}_{0}-\boldsymbol{k}_{a}$ is the momentum transfer. The differential cross section is

$$
\frac{d \sigma}{d \hat{\boldsymbol{k}}_{a}}=\left|f\left(\hat{\boldsymbol{k}}_{a}\right)\right|^{2}
$$

where the scattering amplitude $f$ in Eq. (4) is the Fourier transform of the target charge density in the case of elastic scattering. Therefore, the target charge distributions can be retrieved by analyzing the measured differential cross sections. ${ }^{3,20}$ This charge-density interpretation of the diffraction cross section is attractive, because it provides a physical picture of the target structure.

If the target electronic state is non-stationary, one might be tempted to generalize this interpretation to the case of a time-dependent target electron density $\rho(\boldsymbol{x} ; t)$. Moreover, if the duration of the incident electron pulse is much shorter than the time scale of the target electronic motion, the latter can be treated as frozen in time during the collision process. Thus the target charge density at the moment of collision is recorded in the differential cross section. Movies exhibiting the transient target electronic structure in a reaction can be obtained from a series of such diffraction images obtained by varying the time delay of the electron probe pulse relative to the initiation of the reaction.

\subsection{Simulations}

We adopted the above theory to demonstrate the capability of attosecond electron pulses to image electron dynamics in atoms and molecules. ${ }^{16}$ In the atomic case the target is a coherent superposition state produced by equally superposing the $3 p$ and $4 p$ states of the $\mathrm{H}$ atom. The proposed pump method is detailed in that reference. ${ }^{16}$ The wave function of the coherent state is

$$
\psi_{1}(t)=\frac{1}{\sqrt{2}}\left(\phi_{3 p} e^{-i \omega_{3 p} t}+\phi_{4 p} e^{-i \omega_{4 p} t}\right),
$$

where $\phi_{n p}$ is the eigenstate $n p$ of the $\mathrm{H}$ atom with energy $\omega_{n}$. The electronic motion of this target state exhibits a "breathing mode" (see the left column of Fig. 2) with a beat period T $=2 \pi /\left(\omega_{4 p}-\omega_{3 p}\right)=6.25 \mathrm{fs}\left(1 \mathrm{fs}=10^{-15}\right.$ second). At time zero the electron density is compact around the nucleus, but with increasing time it expands, reaching its maximum radius at $t=\mathrm{T} / 2$, before receding back to the same density at time $t=\mathrm{T}$ as at time zero. Since the constituent eigenstates have the same parity, the electronic motion is symmetric at all times.

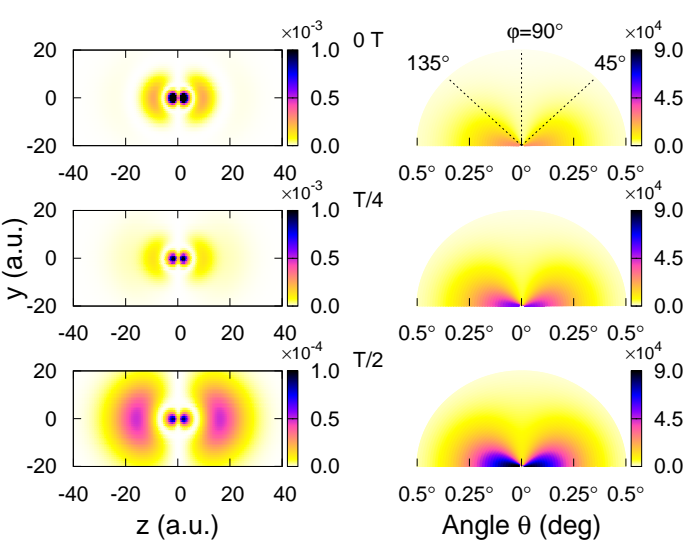

Figure 2: The electron density of the coherent superposition state (6) of the $\mathrm{H}$ atom in the $y-z$ plane as a function of time (left column) and the corresponding differential cross section (right column) of a 258 as (FWHM) electron pulse scattered from that time-dependent state. ${ }^{16}$ Only upper diffraction images are shown owing to symmetry. The coherent state is produced by equally superposing $3 p$ and $4 p$ states of $\mathrm{H}$ atom; it manifests a breathing mode of electronic motion. The beating period is $T=6.25 \mathrm{fs}$. The energy of the projectile electron is $10 \mathrm{keV}$, and its angular divergence is $10^{-3} \mathrm{rad}$. See Figure 1 for the definition of the scattering angle $\theta$ and azimuthal angle $\varphi$. 
The kinetic energy of the ultrafast projectile electron is $10 \mathrm{keV}$ in our simulation, which is an attosecond electron pulse energy considered by Baum and Zewail. ${ }^{11}$ The right column of Figure 2 shows the differential cross section for three different pump-probe delay times: $t_{d}=0 \mathrm{~T}, \mathrm{~T} / 4$, and $\mathrm{T} / 2$. We have taken into account the momentum profile of the projectile electron, which is modeled by a Gaussian distribution, by convoluting the differential cross section in Eq.(5) with the momentum profile. The longitudinal and the transverse widths of the incident electron pulse momentum distribution are set by the pulse duration and the beam angular divergence, respectively. The FWHM duration is 258 as $\left(1 \mathrm{as}=10^{-3} \mathrm{fs}\right)$, and the angular divergence is $10^{-3} \mathrm{rad}$. in our simulations. One sees clearly that the differential cross section varies as the delay time changes, which indicates the capability of an ultrafast electron pulse to image electronic motion. The scattering intensity increases with the spreading of the charge density. In other words, the size of the target is reflected in the scattering pattern. The symmetric diffraction images indicate that the motion is symmetric.

For the molecular case we considered a coherent superposition of the two lowest electronic states $\left(\sigma_{g} 1 s\right.$ and $\left.\sigma_{u} 1 s\right)$ of the tritium molecular ion $\left(\mathrm{T}_{2}^{+}\right)$as the target. ${ }^{16,21,22}$ We substituted the tritium isotope of the hydrogen molecular ion in order to reduce effects of nuclear motion on the coherence of the electronic state. Contrary to the atomic case, $\sigma_{g} 1 s$ and $\sigma_{u} 1 s$ have opposite parity; accordingly, the electron density shows an asymmetric motion, hopping between the two nuclei (left column of Fig.3). In addition to the electronic state, the nuclear degrees of freedom have to be considered in order to simulate the scattering intensities. The bond length is chosen to be large ${ }^{21} R=6$ a.u. ( 1 a.u. $=0.529 \AA$ ), because the potential energy surface is then relatively flat and the energy difference between the electronic states is small. The period $\mathrm{T}$ of the charge oscillation is about $7.1 \mathrm{fs}$. The bond length distribution is assumed to be Gaussian with a 0.71 a.u. width (FWHM). The tritium molecular ion is assumed to be aligned with an angular distribution modeled by $\cos ^{12} \theta_{\text {mol }}$, which gives a half-width $19.3^{\circ}$. The electronic wave function is approximated by a linear combination of atomic orbitals. At large bond lengths the other excited levels of $\mathrm{T}_{2}^{+}$are far from the two lowest levels, indicating that there is little contribution from excited atomic states to the two molecular orbitals we consider. Therefore, only the $1 s$ orbital of the $\mathrm{H}$ atom is used to construct the molecular orbitals.

The differential cross sections are shown on the right in Figure 3 as a function of pump-probe delay time. The diffraction images also exhibit a time dependence as the delay time varies. When the electron density is delocalized and equally populated on both nuclei $(t=\mathrm{T} / 4$, middle panel), the differential cross section exhibits interference fringes at $\theta=1.25^{\circ}$. This is because at $\mathrm{T} / 4$ the delocalization of the charge density behaves like a Young's double slit for the projectile electron. Therefore, since the projectile electron scatters from the charge
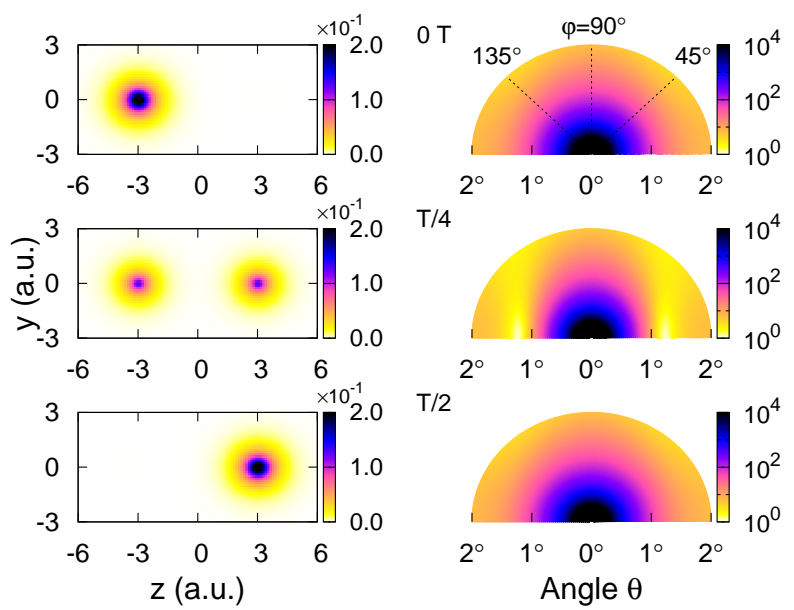

Figure 3: The electron density of a coherent state (an equal superposition of $\sigma_{g} 1 s$ and $\sigma_{u} 1 s$ ) of $\mathrm{T}_{2}^{+}$as a function of time (left column) and the corresponding differential cross sections for scattering of a 258 as electron pulse (right column). ${ }^{16}$ The target coherent state exhibits hopping of the electron between the two nuclei. The molecular bond length distribution is modeled by a Gaussian distribution centered at 6 a.u. with a 0.71 a.u. width (FWHM). The internuclear angular distribution is chosen to be $\cos ^{12} \theta_{\text {mol }}$ such that the half width is $19.3^{\circ}$. 
density at each nuclear site, the scattering amplitudes from each site interfere, causing the interference fringe pattern. (No interference is observed at the other two delay times, when the electron is localized about one or the other nucleus.) Information on the bond length can be obtained by analyzing the angular spacing of the interference pattern. ${ }^{23}$ Note also that the diffraction images at delay times $0 \mathrm{~T}$ and $\mathrm{T} / 2$ are identical. In other words, the diffraction images cannot distinguish on which nucleus the localized electron resides.

\section{ULTRAFAST ELECTRON DIFFRACTION AS COHERENT SCATTERING}

Although the treatment of ultrafast electron diffraction as potential scattering shows positive results for the capability of ultrafast electrons to image target electronic motion, the extension of the charge-density interpretation to time-dependent target states raises some significant issues. First, the meaning of elastic scattering from a non-stationary state is unclear. Second, if the target is regarded as a potential, the entanglement of the scattered electron with the target electrons is neglected. In addition, Dixit et al. recently showed counterintuitive results for X-ray imaging of a non-stationary electronic state. ${ }^{24}$ They simulated the diffraction image of a 1 fs X-ray pulse from a non-stationary state of the $\mathrm{H}$ atom. They found that the time-dependent charge densities are not the quantities imaged in such experiments. They also found that the contributions from radiation damage to the coherent target electronic state (due to inelastic scattering processes) significantly alters the diffraction pattern. In the following we present a time-dependent scattering theory specialized to the case of scattering of an ultrafast electron pulse from a coherent superposition state of a target atom. ${ }^{18}$

\subsection{Theory}

Consider the scattering of an electron from a target $M$ :

$$
e^{-}\left(\boldsymbol{k}_{0}\right)+M\left(\boldsymbol{k}_{1}, n\right) \rightarrow e^{-}\left(\boldsymbol{k}_{a}\right)+M\left(\boldsymbol{k}_{c}, m\right),
$$

where $\boldsymbol{k}_{0}\left(\boldsymbol{k}_{a}\right)$ and $\boldsymbol{k}_{1}\left(\boldsymbol{k}_{c}\right)$ are the respective momenta of the projectile electron and the target before (after) the collision, and $n$ and $m$ specify the initial and final states of the target. The target has some time-dependent electronic structure to be probed.

The main idea of the theory is to construct a coherent wave function comprising both the wave packet of the projectile electron and that of the coherent state of the target at time zero, and then letting the wave function propagate in time. ${ }^{25}$ The transition probabilities are calculated using such a time-dependent wave function. Specifically, let $\psi_{n}^{(+)}$be an eigenstate of the collision system satisfying outgoing wave boundary conditions. The coherent wave function at time zero is expanded in terms of $\psi_{n}^{(+)}$using the wave packet integrals

$$
\psi_{\mathrm{coh}}^{(+)}=\int d \boldsymbol{k}_{0} d \boldsymbol{k}_{1} a_{0}\left(\boldsymbol{k}_{0}\right) a_{1}\left(\boldsymbol{k}_{1}\right) \sum_{n} C_{n} \psi_{n}^{(+)}
$$

where $a_{0}\left(\boldsymbol{k}_{0}\right)$ and $a_{1}\left(\boldsymbol{k}_{1}\right)$ are the respective momentum amplitudes of the projectile electron and the target, and $C_{n}$ is the amplitude of the target internal state $n$. The wave function at later times can be obtained by applying the time evolution operator to $\psi_{\text {coh }}^{(+)}$. The transition probability $\mathscr{P}$ is obtained by summing over all channels measured in the experiment. For ultrafast electron diffraction, only the outgoing direction of the scattered electron is usually measured and the final state $m$ of the target is not usually measured. Therefore inelastic channels representing electron-induced target transitions must be included in $\mathscr{P}$.

According to our theoretical analysis ${ }^{18}$ the preferable conditions for imaging electron dynamics are that the transition probability $\mathscr{P}$ can be factored into two parts: one for the projectile electron and the other for the coherent target. In other words, the properties of the projectile electron and the target coherent state are decoupled, and the transition probability essentially reflects the target properties. In order to achieve this, we find that the projectile electron should have a large kinetic energy and a narrow momentum distribution (i.e., $\left.\Delta k_{0} / k_{0} \ll 1\right)$, so that the transition probability is insensitive to the variation of the momentum components that are integrated over within the wave packet integrals in Eq. (7). Secondly, compared to the time scale of 
the target electronic motion, the duration of the pulse has to be short enough to provide sufficient temporal resolution. Under these conditions, one can show that

$$
\frac{d \mathscr{P}}{d \hat{\boldsymbol{k}}_{a}} \propto \sum_{m}\left|\sum_{n} C_{n} T_{m n}\right|^{2}
$$

where $d \mathscr{P} / d \hat{\boldsymbol{k}}_{a}$ is the differential probability representing the scattering intensity, ${ }^{24}$ and $T_{m n}$ is the transition matrix from the initial target state $n$ to the final target state $m$.

The amplitudes $C_{n}$ of the internal target states $n$ depend on the details of how the initial target coherent state is produced. Because of velocity mismatch between the optical pump pulse and the electronic probe pulse, $C_{n}$ in general depends on the position of the target in a gas ensemble. However, this effect can be mitigated by specially designed pump pulses. ${ }^{6,26}$ Hence, we neglect such effects and set

$$
C_{n}=c_{n} e^{-i \omega_{n} t_{d}}
$$

where $t_{d}$ is the pump-probe delay time, and $c_{n}$ is the amplitude of state $n$ at the moment the coherent state is produced. Combining Eqs. (8) and (9), one has

$$
\frac{d \mathscr{P}}{d \hat{\boldsymbol{k}}_{a}} \propto \sum_{m}\left|\sum_{n} c_{n} T_{m n} e^{-i \omega_{n} t_{d}}\right|^{2} .
$$

This result can be seen as a direct generalization of the conventional scattering theory from stationary states to the case of time-resolved measurements. Equation (10) shows that the differential probability $d \mathscr{P} / d \hat{\boldsymbol{k}}_{a}$ is an incoherent sum over final states $m$ within the resolution of the detector, where each contribution to a particular final state $m$ is given by a coherent sum over the scattering matrices (or the scattering amplitudes) from the constituent eigenstates (indexed by $n$ ) to that final state. By varying the pump-probe delay time $t_{d}$, time-resolved electronic motion can be studied from a series of such scattering images.

We have applied the above theory to two different modes of electronic motion in atoms: breathing and wiggling. Here we summarize the main results of the simulations. For the breathing mode the same constituent states $(3 p$ and $4 p)$ are used as in the $\mathrm{H}$ atom case presented in Sec. 2, while for the wiggling mode, an equal superposition of the $3 d$ and $4 f$ states of the $\mathrm{H}$ atom is employed. ${ }^{24}$ The electron charge density of the wiggling mode oscillates from one side of the nucleus to the other, owing to the opposite parities of the $3 d$ and $4 f$ states (which is similar to the behavior of the charge density for the superposed opposite parity molecular states of the $\mathrm{T}_{2}^{+}$molecular ion considered in Sec. 2). We find that the scattering patterns indeed reflect the electronic motion as the pump-probe delay time is varied in both cases, even though an infinite number of inelastic channels were included in calculating the differential probabilities. Therefore, these results confirm the capability of ultrafast electron pulses to provide time-resolved images of electronic structures. Moreover, the ultrafast electron pulses are able to differentiate different types of electronic motions owing to the different symmetries of the scattering patterns. Nevertheless, we find that the inelastic channels make significant contributions in the forward direction, which makes it difficult to retrieve the target electronic charge density.

\section{TIME-RESOLVED ULTRAFAST ELECTRON MOMENTUM SPECTROSCOPY}

In addition to imaging electron dynamics in the spatial domain, we have also investigated the possibility of mapping the momentum densities of coherent target states by ultrafast $(e, 2 e)$ momentum spectroscopy. ${ }^{17}$ It is well known that $(e, 2 e)$ momentum spectroscopy can measure the momentum profiles of stationary states in atoms and molecules. ${ }^{27-30}$ In contrast to electron diffraction experiments, $(e, 2 e)$ momentum spectroscopy considers the impact ionization of a target electron by a fast incident electron, both of which (i.e., both the scattered and the ejected electrons) are measured in coincidence. The experimental conditions are arranged so as to be close to the Bethe ridge ${ }^{31}$ of the collision, so that the projectile electron essentially collides with the ejected electron, and the nuclei and the other target electrons behave as spectators to the process. In this case one can measure the momentum of the target electron from the momenta of the scattered and ejected electrons using the conservation of momentum in this binary-like collision. The symmetric-nonplanar setup (see Fig.4) 


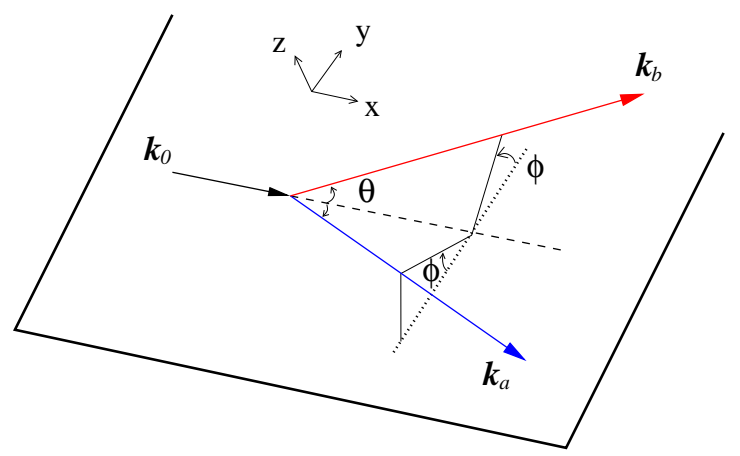

Figure 4: Schematic setup of time-resolved ultrafast $(e, 2 e)$ momentum spectroscopy. ${ }^{17}$ The symmetricnoncoplanar setup is chosen in order to image the momentum density of the coherent electronic state of the target. In this setup the momentum density of the target electron can be measured by varying the detection angle $\phi$ of the two outgoing electrons. The momenta of the projectile, scattered, and ejected electrons are labelled by $\boldsymbol{k}_{0}, \boldsymbol{k}_{a}$, and $\boldsymbol{k}_{b}$, respectively.

is chosen in our study, because for that case the measured signals are directly proportional to the momentum density of the ejected electron. In this setup, the experiment selects $(e, 2 e)$ processes in which the two outgoing electrons equally share the available kinetic energy. The outgoing directions of the scattered and ejected electrons are symmetric but are not in the same plane. The momentum density of the target electron along the $z$ axis can be measured by varying the azimuth angle $\phi$. The $z$ component of the momentum of the target electron, $q_{z}$, and the azimuth angle $\phi$ are related by

$$
q_{z}=\sqrt{2 E_{0}} \sin \frac{\phi}{2}
$$

where is $E_{0}$ is the kinetic energy of the incident projectile electron and where $E_{0} \gg \omega_{n}$ has been assumed.

There are several advantages that $(e, 2 e)$ momentum spectroscopy provides for studying electron dynamics. First, owing to the kinematically-complete measurements, the reaction channels can be analyzed, isolating the electrons participating in the reactions of interest. Second, under appropriate conditions, the measured signals are directly proportional to the momentum density of the ejected electron. Hence, no complicated algorithm is required to retrieve information on the target electronic motion, thus reducing ambiguities. Third, the $(e, 2 e)$ method is sensitive to the momentum density of the valence electrons, which have the most important role in chemical reactions. ${ }^{30}$ Fourth, the symmetry of the target electron charge density is preserved in momentum space $^{28}$ therefore, the symmetry of the system can be easily determined.

\subsection{Theory}

Consider the $(e, 2 e)$ process

$$
e^{-}\left(\boldsymbol{k}_{0}\right)+M\left(\boldsymbol{k}_{1}, n\right) \rightarrow e^{-}\left(\boldsymbol{k}_{a}\right)+e^{-}\left(\boldsymbol{k}_{b}\right)+M^{+}\left(\boldsymbol{k}_{c}, m\right),
$$

where $\boldsymbol{k}_{b}$ is the momentum of the ejected electron, and $m$ here specifies the state of the residual ion. The transition probability in the $(e, 2 e)$ process can be calculated using a coherent wave packet as was done in Sec. 3 for the case of ultrafast electron diffraction. However, the final states in the transition matrix $T_{m n}$ are changed accordingly to take into account the ionization process. Since the experimental setup selects binarylike collisions, the evaluation of $T_{m n}$ is greatly simplified. The central approximation is the plane-wave impulse approximation $^{27,28}$ in which the wave functions for the incoming and outgoing electrons are represented by plane waves and the many-particle transition matrix $T_{m n}$ is replaced by a two-particle one to describe the binary-like collision. One obtains then

$$
T_{m n} \propto \int d \boldsymbol{x}_{b} e^{i \boldsymbol{q} \cdot \boldsymbol{x}_{b}}\left\langle\psi_{m} \mid \psi_{n}\right\rangle
$$


where $\boldsymbol{x}_{b}$ is the coordinate of the ejected electron, $\boldsymbol{q} \equiv \boldsymbol{k}_{a}+\boldsymbol{k}_{b}-\boldsymbol{k}_{0}$ is the momentum of the target electron, and $\psi_{n}$ and $\psi_{m}$ are the eigenstates of the target and the residual ion, respectively. Equation (12) shows that $T_{m n}$ is proportional to the Fourier transform of the target-ion overlap. ${ }^{28}$ Furthermore, because of the fast ejected electron, the interaction time between the ejected electron and the residual ion is short, and the occupied states of the residual ion are identical to those of the neutral state. Therefore, the frozen-core Hartree-Fock approximation is assumed, so that the target-ion overlap is proportional to the orbital of the ejected electron, $\phi_{n}$. Finally, in order to resolve the time-dependent momentum density, the requirements on factorization and pulse duration are the same as for the case of ultrafast electron diffraction. Incorporating these assumptions, one obtains for the triple differential probabilities measured in experiments

$$
\frac{d^{3} \mathscr{P}}{d E_{a} d \hat{\boldsymbol{k}}_{a} d \hat{\boldsymbol{k}}_{b}} \propto\left|\sum_{n} c_{n} \phi_{n}(\boldsymbol{q}) e^{-i \omega_{n} t_{d}}\right|^{2}
$$

Note that the right-hand side of Eq. (13) is the momentum density of the ejected electron at the moment of impact ionization, $t_{d}$.

\subsection{Simulations}

The first example is to image the time-dependent momentum density of the breathing mode of a coherent superposition of the $3 p$ and $4 p$ states of the $\mathrm{H}$ atom. The momentum densities of the coherent state in the $y$ - $z$ plane as a function of time are shown in Fig. 5(b). The momentum density shows an opposite temporal behavior to that of its electron density in the spatial domain [cf. the left column of Fig. 2]. At time zero the momentum density shows a broad distribution, indicating that the coherent state has high momentum components (or high kinetic energy), whereas the distribution shrinks to a more compact form for later times in the first half period of motion. This behavior can be understood by inspecting the corresponding radial part of the electron density and the atomic potential shown respectively in the upper and lower parts of Fig. 5(a). The radial density $R_{3 p+4 p}$ at $t=0$ is narrow and localized about the nucleus [solid red curve of Fig. 5(a)], where the Coulomb potential is deepest; accordingly, the coherent state has large kinetic energy (and large momentum components), and hence a broad distribution of momentum density. On the other hand, when $t=\mathrm{T} / 2$ the charge density [dotted blue curve in Fig. 5(a)] extends over the region where the Coulomb potential is shallow, so the momentum distribution is concentrated in the region of smaller momentum components, as shown in the bottom panel of Fig. 5(b).

Since the excited $3 p$ and $4 p$ states have large average radii, the momentum density of the coherent superposition state is found to be $\lesssim 0.3$ a.u. $\left(1\right.$ a.u. $\left.=11.9 \AA^{-1}\right)$. Therefore, the momentum resolution and the

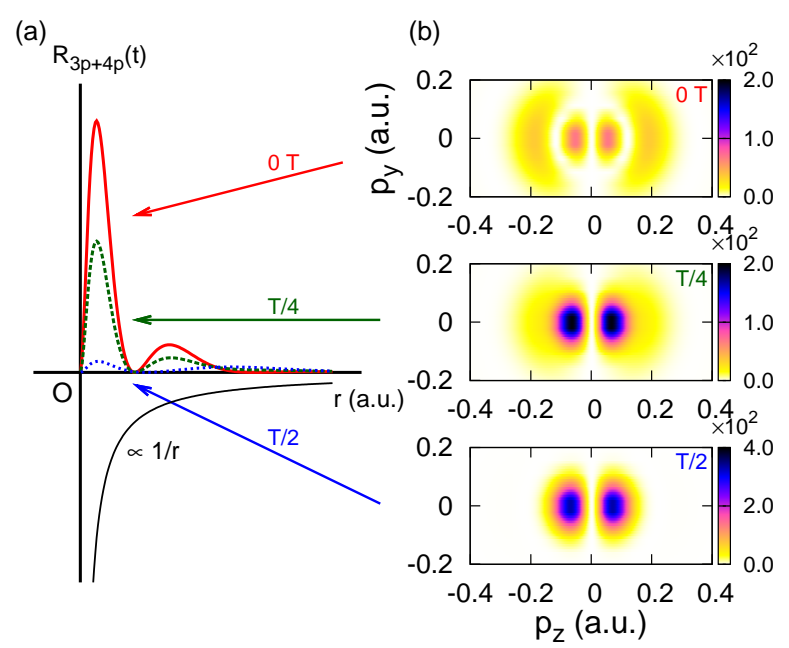

Figure 5: (a) Upper panel: The radial probability density of the breathing mode of a coherent superposition of the $3 p$ and $4 p$ states of the $\mathrm{H}$ atom at three different times, $t=0, \mathrm{~T} / 4$, and $\mathrm{T} / 2$, where $\mathrm{T}$ is the beat period of the coherent target state; Lower panel: the Coulomb potential. (b) The momentum density in the $y$ - $z$ plane at the same three times; note that the vertical scale of the bottom panel differs from that of the top two panels. 


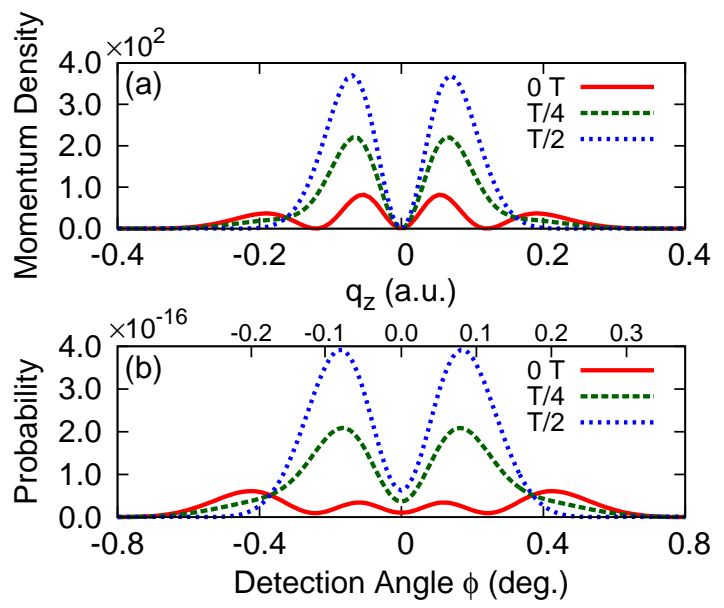

Figure 6: (a) The momentum densities of the breathing mode of electronic motion of a coherent superposition of the $3 p$ and $4 p$ states of the $\mathrm{H}$ atom along the $z$ axis at three different times, $t=0, \mathrm{~T} / 4$, and $\mathrm{T} / 2$, where $\mathrm{T}$ is the beat period of the coherent target state. (b) The measured probabilities integrated over the distributions of the experimental parameters at the same three pump-probe delay times. ${ }^{17}$

momentum profile of the projectile electron have to be considered for this case involving such small momenta, because the convolution of the scattering results with the projectile and target momentum distributions may affect the measured momentum density. ${ }^{32}$ The projectile electron is modeled in the same manner as in the electron diffraction simulations in Secs. 2 and 3. Its pulse duration is 100 as (FWHM) and its angular divergence is $10^{-3} \mathrm{rad}$. In order to study the effect of the width of the detection window on the resolution of the measured momentum profile, we chose the detection widow to be $\theta \times \phi=0.2^{\circ} \times 0.2^{\circ}$. As for the simulations of ultrafast electron diffraction from the $\mathrm{H}$ atom, no factorization is assumed in calculating the transition probabilities. The transition probability (convoluted with the detection window) along the $z$ axis together with the corresponding momentum density are shown as a function of the pump-probe delay time in Fig. 6. One sees that the transition probabilities agree well with the momentum densities of the coherent state. The non-zero minimum of the central valley $\left(\phi=0^{\circ}\right)$ in Fig. 6 (b) is due to the finite detection window. ${ }^{32}$

The second example we investigate is that of an equal superposition of the $\sigma_{g} 1 s$ and $\sigma_{u} 1 s$ states of the $\mathrm{H}_{2}^{+}$ molecular ion, which involves the hopping of the electron between the two nuclei once every half beat period $\mathrm{T}$. The momentum densities along the molecular axis as a function of time are shown in Figs. 7(a) and (b), where the inset figures show the corresponding electron densities along that axis. The bond length is set at $R=6$ a.u. The asymmetric electronic motion is also manifested in its momentum density. At time $t=0$, the electron charge density resides on the left nucleus (solid black line), so the electron's momentum density is symmetric and resembles that of the $1 s$ stationary state of the $\mathrm{H}$ atom. As the electron hops to the right nucleus at $t=\mathrm{T} / 4$, one sees that the momentum density shifts toward positive momentum components (dotted red line). At $t=\mathrm{T} / 2$ the electron charge density is located at the right nucleus and the momentum density is once again symmetric. When the electron moves back to the left nucleus at $t=3 \mathrm{~T} / 4$, the momentum density shifts accordingly toward negative momentum components. In order to observe the asymmetric electronic motion, the $\mathrm{H}_{2}^{+}$molecular ion must be oriented. Here we assumed that the $\mathrm{H}_{2}^{+}$molecular ion is oriented perpendicular to the incident direction of the ultrafast probe electron with a distribution $\left(\propto \cos ^{6} \theta_{\mathrm{mol}}+\alpha \cos ^{7} \theta_{\mathrm{mol}}\right)$ such that $\left\langle\cos ^{2} \theta_{\mathrm{mol}}\right\rangle=0.78$ and $\left\langle\cos \theta_{\text {mol }}\right\rangle=0.39$. The molecular bond length is assumed to be Gaussian with a 0.3 width (FWHM). Since the target momentum distribution in this case has larger momentum components, which are easier to resolve, it was not necessary to integrate the triple differential probability over the experimental detection window. The results are shown in Fig. 7(c). The results do show the target time dependence; however, the triple differential probability only qualitatively agrees with the momentum density. We find that the transition probability is quite sensitive to the angular distribution of the $\mathrm{H}_{2}^{+}$molecular axis. Better agreement can be obtained for a higher degree of molecular orientation. 

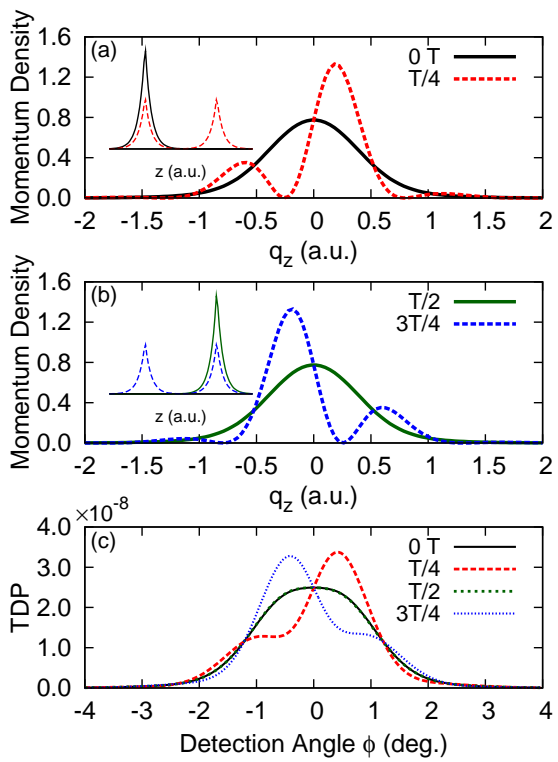

Figure 7: (a-b) The momentum density of an equal superposition of the $\sigma_{g} 1 s$ and $\sigma_{u} 1 s$ states of the $\mathrm{H}_{2}^{+}$molecular ion along the molecular axis at four different times, $t=0, \mathrm{~T} / 4, \mathrm{~T} / 2$, and $3 \mathrm{~T} / 4$, where $\mathrm{T}$ is the beat period of the coherent target state. The inset figures show the corresponding electron charge densities. (c) The corresponding triple differential probability (TDP) at the same four values of pump-probe delay times. ${ }^{17}$ The $\mathrm{H}_{2}^{+}$molecular ion is assumed to be oriented such that $\left\langle\cos ^{2} \theta_{\mathrm{mol}}\right\rangle=0.78$ and $\left\langle\cos \theta_{\mathrm{mol}}\right\rangle=0.39$. The bond length is modeled by a Gaussian distribution centered at $R=6$ a.u. with FWHM of 0.3 a.u.

\section{CONCLUSION}

In conclusion, we have investigated three possibilities for direct imaging of electronic motion using ultrafast (attosecond) electron pulses. Two different theories for modeling ultrafast electron diffraction from coherent states of target atoms and molecules are presented. In the first approach the target is modeled as a time-dependent effective potential, whereas in the second approach the target coherent state is treated rigorously in terms of its wave function. Although the first approach provides a clear and physical picture describing the diffraction process, it cannot fully take account of the diffraction process. To achieve time-resolved images, we find that both the transverse and the longitudinal coherence of the projectile electron are important. ${ }^{33}$ The transverse coherence determines the spatial resolution for resolving target electronic structures, while the longitudinal coherence limits the temporal resolution. Under appropriate conditions, such as for the case of ultrafast electron impact ionization - the $(\mathrm{e}, 2 \mathrm{e})$ process - in the symmetric-noncoplanar experimental configuration shown in Fig. 4, the measured signals directly provide time-dependent properties of the target states. With the anticipated experimental advances of ultrafast electron pulse production and measurement capabilities, the direct investigation of the role of electrons in reaction processes as a function of time will provide valuable information for both understanding and controlling those reactions.

\section{ACKNOWLEDGMENTS}

This work is supported in part by AFOSR Award No. FA9550-12-1-0149 and by NSF Grant No. PHYS-1208059. Some of our calculations were carried out at the Holland Computing Center of the University of Nebraska.

\section{REFERENCES}

[1] Kling, M. F. and Vrakking, M. J., "Attosecond electron dynamics," Annu. Rev. Phys. Chem. 59, 463-492 (2008).

[2] Krausz, F. and Ivanov, M., "Attosecond physics," Rev. Mod. Phys. 81, 163-234 (2009). 
[3] Bonham, R. A. and Fink, M., High Energy Electron Scattering, Van Nostrand Reinhold Company, New York (1974).

[4] Zewail, A. H., "4D ultrafast electron diffraction, crystallography, and microscopy," Annu. Rev. Phys. Chem. 57, 65-103 (2006).

[5] Zewail, A. H., "Four-dimensional electron microscopy," Science 328, 187-193 (2010).

[6] Sciaini, G. and Miller, R. J. D., "Femtosecond electron diffraction: heralding the era of atomically resolved dynamics," Rep. Prog. Phys. 74, 096101 (2011).

[7] Ihee, H., Lobastov, V. A., Gomez, U. M., Goodson, B. M., Srinivasan, R., Ruan, C.-Y., and Zewail, A. H., "Direct imaging of transient molecular structures with ultrafast diffraction," Science 291, 458 (2001).

[8] Flannigan, D. J. and Zewail, A. H., "4D electron microscopy: Principles and applications," Acc. Chem. Res. 45, 1828-1839 (2012).

[9] Aidelsburger, M., Kirchner, F. O., Krausz, F., and Baum, P., "Single electron pulses for ultrafast diffraction," Proc. Natl. Acad. Sci. U.S.A. 107, 19714-19719 (2010).

[10] Fill, E., Veisz, L., Apolonski, A., and Krausz, F., "Sub-fs electron pulses for ultrafast electron diffraction," New J. Phys. 8, 272 (2006).

[11] Baum, P. and Zewail, A. H., "Attosecond electron pulses for 4D diffraction and microscopy," Proc. Natl. Acad. Sci. U.S.A. 104, 18409-18414 (2007).

[12] Hilbert, S. A., Uiterwaal, C., Barwick, B., Batelaan, H., and Zewail, A. H., "Temporal lenses for attosecond and femtosecond electron pulses," Proc. Natl. Acad. Sci. U.S.A. 106, 10558-10563 (2009).

[13] Gliserin, A., Apolonski, A., Krausz, F., and Baum, P., "Compression of single-electron pulses with a microwave cavity," New J. Phys. 14, 073055 (2012).

[14] Baum, P. and Zewail, A. H., " $4 \mathrm{D}$ attosecond imaging with free electrons: Diffraction methods and potential applications," Chem. Phys. 366, 2-8 (2009).

[15] Baum, P., Manz, J., and Schild, A., "Quantum model simulations of attosecond electron diffraction," Sci. China Phys. Mech. Astron. 53, 987-1004 (2010).

[16] Shao, H.-C. and Starace, A. F., "Detecting electron motion in atoms and molecules," Phys. Rev. Lett. 105, 263201 (2010).

[17] Shao, H.-C. and Starace, A. F., "Time-resolved ultrafast electron $(e, 2 e)$ momentum spectroscopy," Phys. Rev. A 87, 050701(R) (2013).

[18] Shao, H.-C. and Starace, A. F. (to be published).

[19] Bethe, H. A. and Jackiw, R., Intermediate Quantum Mechanics, W. A. Benjamin, Inc., New York, 1968 (second edition).

[20] Spence, J. C. H., "Diffractive (Lensless) Imaging," in Science of Microscopy, Vol. I, Hawkes, P. W. and Spence, J. C. H., eds., 1196-1227, Springer, New York, 2007.

[21] Yudin, G. L., Chelkowski, S., Itatani, J., Bandrauk, A. D., and Corkum, P. B., "Attosecond photoionization of coherently coupled electronic states," Phys. Rev. A 72, 051401(R) (2005).

[22] Bandrauk, A. D., Chelkowski, S., Corkum, P. B., Manz, J., and Yudin, G. L., "Attosecond photoionization of a coherent superposition of bound and dissociative molecular states: effect of nuclear motion," J. Phys. $B$ 42, 134001 (2009).

[23] Srinivasan, R., Lobastov, V. A., Ruan, C.-Y., and Zewail, A. H., "Ultrafast electron diffraction (UED)," Helv. Chim. Acta 86, 1761-1838 (2003).

[24] Dixit, G., Vendrell, O., and Santra, R., "Imaging electronic quantum motion with light," Proc. Natl. Acad. Sci. U.S.A. 109, 11636-11640 (2012).

[25] Robicheaux, F., "Scattering with longitudinally coherent matter beams," Phys. Rev. A 62, 062706 (2000).

[26] Baum, P. and Zewail, A. H., "Breaking resolution limits in ultrafast electron diffraction and microscopy," Proc. Natl. Acad. Sci. U.S.A. 103, 16105-16110 (2006).

[27] McCarthy, I. E. and Weigold, E., "Electron momentum spectroscopy of atoms and molecules," Rep. Prog. Phys. 54, 789-879 (1991).

[28] Coplan, M. A., Moore, J. H., and Doering, J. P., “(e,2e) spectroscopy,” Rev. Mod. Phys. 66, 985-1014 (1994). 
[29] Brion, C. E., Cooper, G., Zheng, Y., Litvinyuk, I. V., and McCarthy, I. E., "Imaging of orbital electron densities by electron momentum spectroscopy - a chemical interpretation of the binary (e,2e) reaction," Chem. Phys. 270, 13-30 (2001).

[30] Takahashi, M., "Looking at molecular orbitals in three-dimensional form: From dream to reality," Bull. Chem. Soc. Jpn. 82, 751-777 (2009).

[31] Inokuti, M., "Inelastic collisions of fast charged particles with atoms and molecules-the Bethe theory revisited," Rev. Mod. Phys. 43, 297-347 (1971).

[32] Duffy, P., Cassida, M. E., Brion, C. E., and Chong, D. P., "Assessment of gaussian-weighted angular resolution functions in the comparison of quantum-mechanically calculated electron momentum distributions with experiment," Chem. Phys. 159, 347-363 (1992).

[33] Baum, P., "On the physics of ultrashort single-electron pulses for time-resolved microscopy and diffraction," Chem. Phys. 423, 55-61(2013). 